EPJ Web of Conferences 32, 04008 (2012)

DOI: $10.1051 /$ epjconf/20123204008

(C) Owned by the authors, published by EDP Sciences, 2012

\title{
Status of Resonant Diplexer Development for high-power ECRH Applications
}

W. Kasparek ${ }^{1}$, B. Plaum ${ }^{1}$, C. Lechte ${ }^{1}$, E. Filipovic ${ }^{1}$, V. Erckmann ${ }^{2}$, G. Grünwald ${ }^{2}$, F. Hollmann ${ }^{2}$, M. Maraschek ${ }^{2}$, G. Michel ${ }^{2}$, F. Monaco ${ }^{2}$, S. Müller ${ }^{2}$, F. Noke ${ }^{2}$, F. Purps ${ }^{2}$, M Schubert $^{2}$, H. Schütz ${ }^{2}$, J. Stober ${ }^{2}$, D. Wagner ${ }^{2}$, R. van den Braber ${ }^{3}$, N. Doelman ${ }^{3}$, E. Fritz ${ }^{3}$, W. Bongers ${ }^{4}$, B. Krijger ${ }^{4}$, M. Petelin ${ }^{5}$, L. Lubyako ${ }^{5}$, A. Bruschi ${ }^{6}$, K. Sakamoto ${ }^{7}$, teams at the contributing institutes, and ASDEX Upgrade Team.

${ }^{1}$ Institut für Plasmaforschung, Universität Stuttgart, D-70569 Stuttgart, Germany

${ }^{2}$ Max-Planck-Institut für Plasmaphysik, EURATOM Association D-17491 Greifswald, and D-85748 Garching, Germany

${ }^{3}$ Department of OptoMechatronics, TNO Technical Sciences, NL-2600 AD, Delft, The Netherlands

${ }^{4}$ Dutch Institute For Fundamental Energy Research, NL-3430 BE Nieuwegein, The Netherlands

${ }^{5}$ Inst. of Applied Physics, Russian Academy of Science, 603950 Nizhny Novgorod, Russia

${ }^{6}$ Istituto di Fisica del Plasma, EURATOM-ENEA-CNR Association, I-20125 Milano, Italy

${ }^{7}$ Japan Atomic Energy Agency (JAEA), 801-1, Mukoyama, Naka, Ibaraki 311-0193, Japan

\begin{abstract}
Characteristics of ring resonator diplexers for high-power ECRH are briefly reviewed. Commissioning experiments performed on ASDEX Upgrade with the diplexer Mk IIa are presented, which demonstrate slow and fast switching of the power between two launchers, and thus the capability for efficient suppression of neoclassical tearing modes and simultaneous central heating of the plasma. The development of the compact diplexer Mk IIIb is discussed, and test results are presented. Finally, an evacuated design for $170 \mathrm{GHz}$ is shown.
\end{abstract}

\section{Introduction}

In the past years, high-power diplexers have become of growing interest for applications in ECRH systems. Different variants [1] have been investigated, and especially ring resonator diplexers [2] have demonstrated high versatility. The example of the $170 \mathrm{GHz} / 24$ MW ITER ECRH system [3], where the power can be switched between the equatorial launcher (EL) and the upper launchers (UL) by waveguide switches, illustrates the many options. If the mechanical switches were replaced by four-port resonant diplexers, the power could be (i) arbitrarily distributed between the launchers according to the physics needs by simply tuning the resonance frequency, while the gyrotrons run continuously. For synchronous NTM stabilization [4], a gyrotron voltage modulation synchronous to the rotation frequency of the islands together with a proper tuning of the diplexer would (ii) strongly amplify the amplitude modulation in the UL and thus yield high stabilization efficiency. In this context, the mode purification characteristics of the resonator could (iii) improve the focussing and reduce the possible beam squint of the UL. The filtering of very-high-order modes would (iv) reduce the thermal loading of the sensitive cuffs of the vacuum windows in the UL as well as in the EL. A 
stray radiation detector in the diplexer would (v) serve as a monitor for mode purity. The isolated input port could be used to (vi) receive ECE radiation from the plasma by sharing the UL with the (forward) ECRH [5]. Eventually, if an upgrade of the ECRH power is needed, these second input ports could be (vii) used to connect further gyrotrons, as the diplexer can operate as power combiner. The drives tuning the resonator mirrors can be easily integrated into the control system, as the gyrotrons can run continuously at any time during the tuning process.

In this paper, basic design features of resonant diplexer are briefly reviewed (chapter 2). The diplexer Mk IIa, which uses a gaussian beam resonator and $\mathrm{HE}_{11}-\mathrm{TEM}_{00}$ mode converters to be compatible with $\mathrm{HE}_{11}$ transmission, was extensively tested at the ECRH system of W7-X. Recently, it was integrated into the ECRH system of ASDEX Upgrade; first experiments are described in chapter 3. Meanwhile, the compact diplexer MC IIIb was built, and tests are presented in chapter 4 . This diplexer version is taken as basis for the construction of the evacuated type MQ IV, which is compatible with the ITER ECRH (chapter 5).

\section{Resonant Diplexers}

Resonant diplexers for high power can be designed as 4-mirror ring resonators, where two of the mirrors are -1 st - order gratings serving as input and output couplers, as sketched in Fig.1a. The basic transmission characteristics [6], namely narrow resonances in output 2 ("resonant channel") periodic with $\mathrm{c} / L$, and broad transmission regions in between ("non-resonant channel"), are determined by the round-trip length $L$ and the coupling efficiency $R_{1}$, as shown in Fig. 1b. Using a control for $L$ by making one of the resonator mirrors movable (linear motion less than one wavelength) [6, 7], the diplexer can be tuned according to the application, especially to point $\mathrm{A}$, where the gyrotron frequency $f_{\mathrm{gyr}}$ is resonant, and point $\mathrm{B}$, where $f_{\mathrm{gyr}}$ corresponds to the slope of the resonance.

Several prototypes for high power at $140 \mathrm{GHz}$ were developed, and two (Mk I and Mk IIa) were tested extensively at the W7-X ECRH system [8]. "Slow" switching of the continuous power from one gyrotron between two output channels by controlled mirror movement, "fast" switching by frequency-shift keying of the gyrotron (using few $\mathrm{kV}$ modulation of the body voltage), and power combination from two gyrotrons was demonstrated [6].
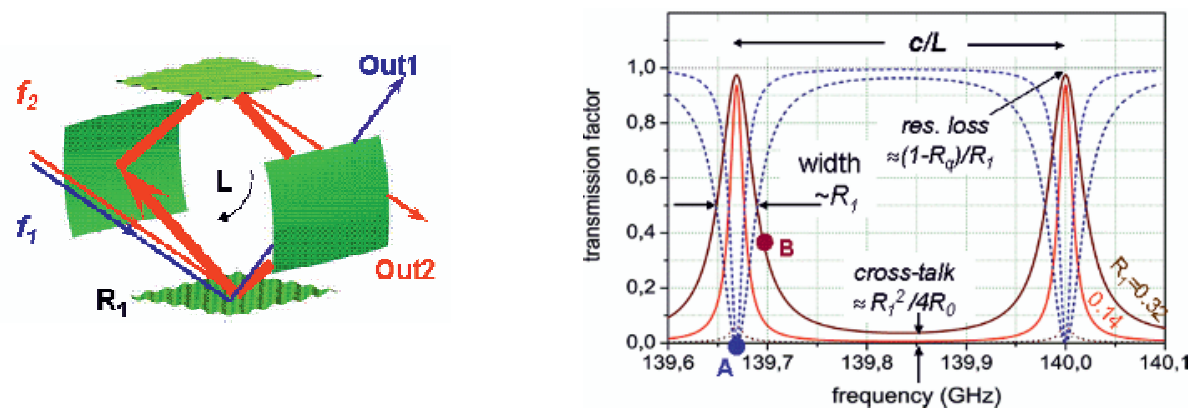

Fig. 1. Principle sketch (left) and transmission functions (right) for the non-resonant (IN - OUT1, dashed lines) and the resonant (IN - OUT2, solid lines) channel. The transmission is mainly determined by the $-1^{\text {st }}$ order efficiency $R_{1}$ of the coupling grating $R_{1}=1-R_{0}$, where $R_{0}$ is the power coefficient for specular reflection. A and B are operation points e.g. for in-line ECE and fast switching, respectively.

\section{ECRH experiments at ASDEX Upgrade}

The Mk IIa was integrated into the corrugated waveguide transmission of the ECRH-2 system at ASDEX upgrade [9], with one input connected to gyrotron Gy2, and with the outputs feeding the launcher L3 via the non-resonant channel, and launcher L1 via the resonant channel. L3 was adjusted for central heating, L1 for off-axis power deposition. To match the polarisation to the launching 
geometry, mitre-bend polarisers were installed behind the diplexer. With this set up, various commissioning experiments were performed in L-mode discharges, which were centrally heated by $0.8 \mathrm{MW}$ ECRH and in addition with $0.5 \mathrm{MW}$ from Gy2. Switching between the launchers $(1.2 \mathrm{~s}$ power on L1, then $0.4 \mathrm{~s}$ power on L3, and again $1.3 \mathrm{~s}$ power to L1) was demonstrated by tuning the diplexer from resonance to non-resonant conditions, and back.

Periodic toggling of the power with $32 \mathrm{~Hz}$ was tested by a $\pm 80 \mu \mathrm{m}$ oscillation of the resonator mirror along the slope of the resonance. Such an experiments could be useful for locking sawteeth to the oscillation frequency, where the power toggles between locations inside and outside the $\mathrm{q}=1$ - surface, and the gyrotron runs $\mathrm{cw}$.

Fast switching as preparation for synchronous NTM stabilization was performed by tuning the diplexer to B (Fig. 1b) and then starting modulation of the acceleration voltage with $\Delta U_{\mathrm{B}}=3.5 \mathrm{kV}$ and $f_{\text {mod }}=25 \mathrm{kHz}$ (which is the maximum NTM frequency at ASDEX Upgrade). With the resulting frequency shift keying of $\Delta f_{\text {mod }}=14 \mathrm{MHz}$, a switching contrast of $70-80 \%$ at the off-axis launcher (where the power variation due to frequency-shift keying is in phase with the gyrotron power variation due to voltage modulation) was measured by oscilloscopes. As the data acquisition at $25 \mathrm{kHz}$ was at its time resolution limit, the same experiment was repeated with $f_{\text {mod }}=200 \mathrm{~Hz}$. This low frequency allows the detection of heat waves generated in the plasma by the power toggling between the launchers. The results are shown in Fig. 2. The signals on the left of Fig. 2 clearly show the inevitable power modulation $\left(1^{\text {st }}\right.$ plot $)$ of about $30 \%$ due to the voltage modulation as well as the resulting frequency modulation $\left(2^{\text {nd }}\right.$ plot $)$.
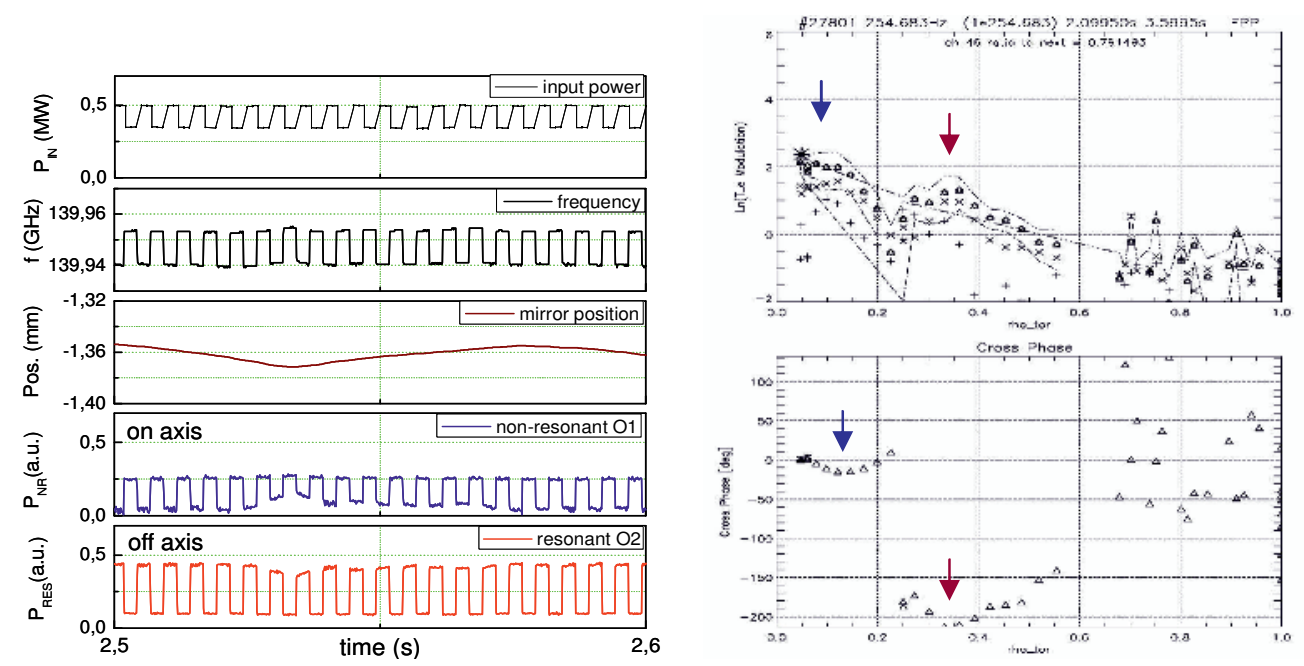

Fig. 2. Left: Input power, gyrotron frequency, mirror position, as well as the power signals for the onaxis and the off-axis launcher, when the gyrotron is square-wave modulated with $\Delta U_{\mathrm{B}}=3.5 \mathrm{kV}$ at $f_{\text {mod }}=200 \mathrm{~Hz}$. Right: Heat wave analysis showing the deposition regions for the on-axis launcher (blue arrows) and the off-axis launcher (red arrows) from amplitude (upper) and phase (lower plot).

The resonator mirror position $\left(3^{\text {rd }}\right.$ plot) is controlled such that maximum power modulation in the off-axis launcher is obtained $\left(5^{\text {th }}\right.$ plot $)$. Note that a complete switching of the power is not possible within the trade-off chosen between minimum power modulation and maximum frequency-shift keying. However, efficiency calculations on synchronous NTM stabilization show that power modulation of $70 \%$ should be sufficient. On the right of Fig. 2 the heat wave analysis from the electron temperature $\left(T_{e}\right)$ profile measured by ECE is shown. From the amplitude of the $T_{e}$ modulation (upper figure) as well as from the minima of the cross phase, two deposition regions at plasma radii of $\rho_{\text {tor }}<0.1$ and $\rho_{\text {tor }} \approx 0.35$ can be clearly identified. The phase difference of $180^{\circ}$ between the two heat waves supports the assumption of the toggling power. 
In-line ECE experiments performed at ASDEX Upgrade are described in a separate paper [10].

\section{Development of compact resonant diplexers}

\subsection{Design of Mk IIIb}

The device Mk IIa was operated with a Gaussian beam resonator, which requires transitions from $\mathrm{HE}_{11}$ to $\mathrm{TEM}_{00}$ at the inputs and outputs. The compact $\mathrm{Mk}$ IIIb $(L=0.906 \mathrm{~m})$ features direct corrugated waveguide input, as ring resonators with phase-reversing mirrors matched to the $\mathrm{HE}_{11}$ fields are used. Fig. 3a shows the amplitude and phase of the field impinging on the resonator mirror MR; the similarity of the surface of MR (in Fig. 3b) and the phase profile is clearly seen. Using double-face matching mirrors (MM) shown in Fig $3 \mathrm{~b}$ and $3 \mathrm{c}$ allows the mounting of one optical element on either side of the diplexer casing, which yields a precise and stable alignment.
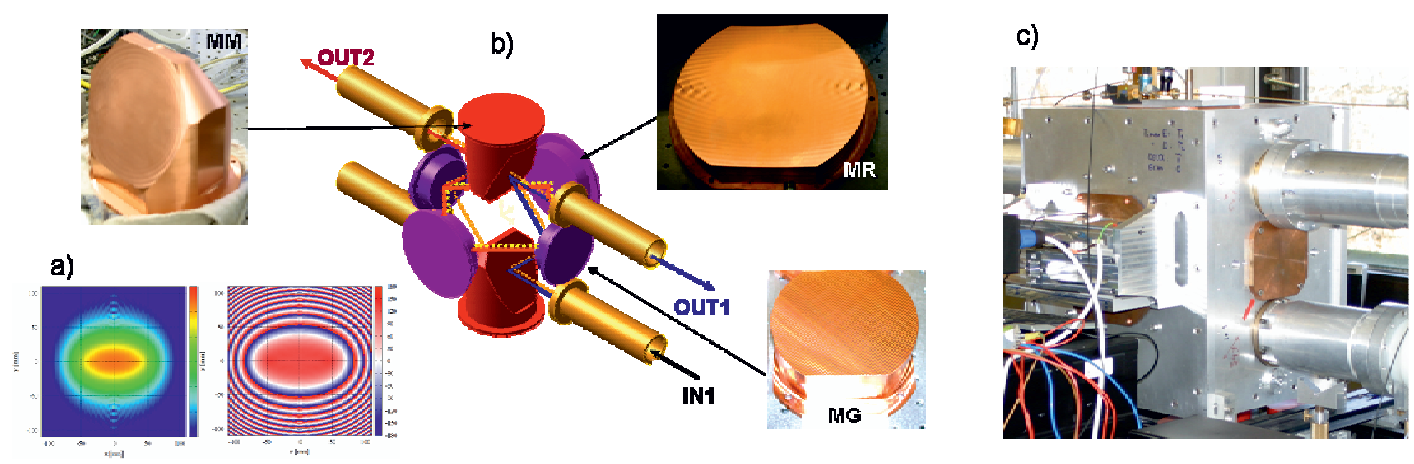

Fig. 3. Design of the resonant diplexer Mk III b. a) Calculated amplitude and phase profile on the resonator mirror. b) Optical set up with pictures of a matching mirrors MM, a coupling grating MG and a resonator mirror MR. c) Test set up of the diplexer with waveguides connected and with the mirror control mounted to one resonator mirror (in the foreground).

\subsection{Low-power test}

The diplexer Mk IIIb was characterized using various coupling gratings. With very shallow gratings, the internal resonator loss could be measured precisely from the unloaded quality factor Q, resulting in an internal round-trip efficiency of $R_{\mathrm{q}}=0.991$. The transmission loss through the non-resonant channel alone was determined by a resonator experiment, where the connecting waveguides were closed by plane reflectors and the gratings were replaced with plane mirrors; from the quality factor of this resonator, an $\mathrm{HE}_{11}$ transmission efficiency of $\eta_{\mathrm{nr}, \mathrm{HE} 11}=0.993$ was obtained. The complete transmission functions were recorded with a tuneable gunn oscillator and a (calorimetric) TK power meter, where the transmitted power in practically all modes is measured. The results for a Mk III version with relatively broad resonances $\left(R_{1}=0.284\right)$ optimized for in-line ECE are plotted in Fig. 4. From the fit of the theory, and including the results given above, the total power transmission efficiencies for the resonant and the non-resonant channel are obtained as $\eta_{\text {r,total }}=0.947$ and $\eta_{\text {nr,total }}=0.97$, respectively. The transmission efficiency in the resonant channel for a pure $\mathrm{HE}_{11}$ mode is $\eta_{\mathrm{r}, \mathrm{HE} 11}=0.963$. All data are summarized in table 1 .

As seen in Fig. 4, the transmission functions are in good agreement with theory, with one exception, namely the depth of the notch at the resonance frequency. Theory predicts a relative depth of $2 \cdot 10^{-4}$, and indeed, typically $10^{-3}$ is measured, when an $\mathrm{HE}_{11}$ receiver is used. The reason for this discrepancy is the limited mode purity of the $\mathrm{HE}_{11}$ mode generator used for the test. Other modes than the $\mathrm{HE}_{11}$ have different resonance frequencies, and thus are mostly transmitted to the non- 
resonant channel. This residual power is a measure for the mode-purity at the input to the diplexer; in the present case this means $4.5 \%$ wrong modes minimum. This feature could be used to optimize the alignment in a transmission system.

Table 1. Summary of transmission characteristics of Mk IIIb.

\begin{tabular}{|c|c|c|c|c|}
\hline & \multicolumn{2}{|c|}{ non-resonant channel } & \multicolumn{2}{c|}{ resonant channel } \\
& value & remark & value & remark \\
\hline$\eta_{\text {HE11 }}$ & $0.993^{ \pm 0.001}$ & resonator experiment & $0.963^{ \pm 0.003}$ & resonator / calorimetry \\
\hline$\eta_{\text {total }}$ & $0.970^{ \pm 0.003}$ & calorimetry & $0.947^{ \pm 0.005}$ & calorimetry \\
\hline cross-talk & $0.026^{ \pm 0.002}$ & according to theory & $0.045^{ \pm 0.005}$ & wrong modes at input \\
\hline
\end{tabular}

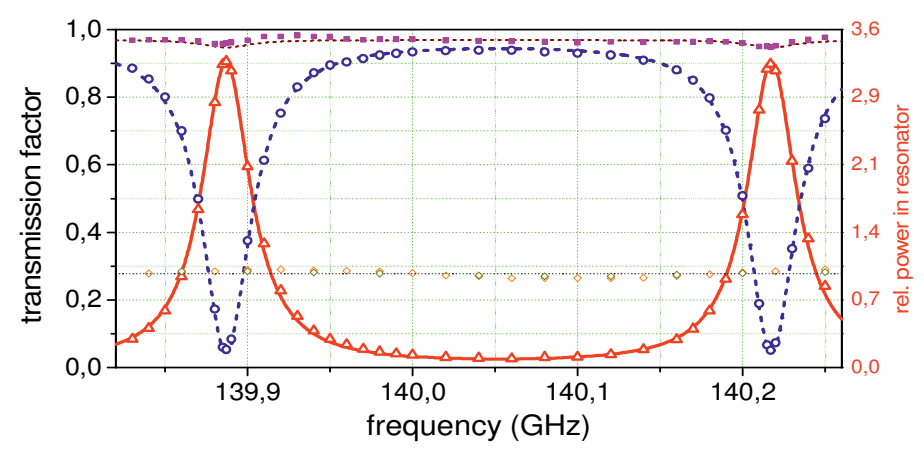

Fig. 4. Power transmission for the diplexer Mk IIIb with gratings with $R_{1}=0.284, L=0.9062 \mathrm{~m}$. symbols: measurement of resonant (triangles), non-resonant (circles), and the sum of both outputs (squares). Lines: Corresponding calc. transmission functions taking into account $4.5 \%$ wrong modes.

On the other hand, the $\mathrm{HE}_{11}$ resonance frequency can be used for mode filtering in the transmission line. This is demonstrated by the extreme example of an " $\mathrm{HE}_{11}$ " input mode with a purity of $48 \%$ only. At the resonant output of $\mathrm{Mk}$ IIIb (equipped with gratings with $R_{1}=0.041$ for narrow resonances), an $\mathrm{HE}_{11}$ with a purity of $92 \%$ is obtained, as shown in Fig. 5. Here, lower-order wrong modes are transmitted to the non-resonant output, very high-order modes are absorbed in the diplexer casing.
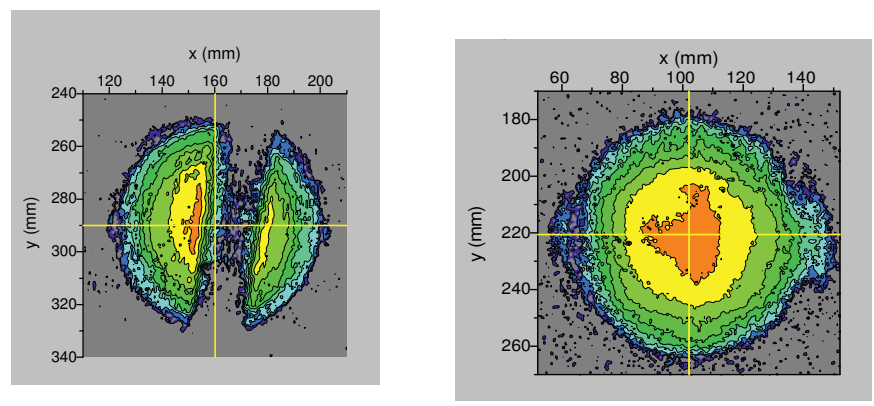

Fig. 5. Test of the mode purification of resonant diplexers. Left: Input pattern of an $\mathrm{HE}_{11}$ mode with artificially reduced purity of $48 \%$. Right: Pattern at the resonant output with mode purity of $92 \%$. 
The isolation of the diplexer was tested by measuring the power in the $\mathrm{HE}_{11}$ mode (i.e. with a matched receiver) in the isolated port for different loads at the outputs. Results similar to those for Mk IIa [11] were obtained. For open (non-reflecting) outputs, a very high isolation of $>65 \mathrm{~dB}$ is obtained. If a $100 \%$ reflection from a plane mirror, i.e. in $\mathrm{HE}_{11}$ mode, occurs, the power received in the isolated port is given by the product of the transmission functions, i.e. in the worst case is only 6 $\mathrm{dB}$ below the input power. In the more practical case, where a reflection (e.g. from the plasma vessel or an arc) occurs in multi-mode (simulated by a $100 \%$ power reflection from a crumpled aluminium foil), the isolation is $\geq 40 \mathrm{~dB}$.

To further improve and simplify the resonator control, a microwave circuit was developed, which generates an error signal from the sum and the difference of directional coupler signals at the outputs of the diplexer. Details on the test of this new control scheme are found in [7].

\section{A compact diplexer for $170 \mathrm{GHz}$}

Based on the Mk III design, a diplexer mock-up which is compatible with the $170 \mathrm{GHz}$ ITER ECRH system is under development. It consists of a vacuum-tight box made from a solid Al block, with precise mounting flanges for mirrors and waveguides. The top plate of the box carries the upper resonator mirror, absorber tubes, and eventually the resonator control, while integrated directional grating couplers deliver the control signals for the drive. The present version features solid $\mathrm{Cu}$ mirrors without cooling; however, cooling could be added later easily without extra vacuum feedthroughs. The present plans aim at fabrication in the second half of 2012; tests shall start end of 2012 at IPF (low-power) and JAEA (high-power).

\section{Summary}

The present paper shows that resonant diplexers for ECRH have reached a high level of development. A broad range of applications like slow and fast switching as well as arbitrary power distribution between launchers, power combination, mode filtering, spurious mode diagnostics, and in-line ECE measurements could be confirmed by experiments. An experiment on synchronous NTM stabilization is in preparation. The compact diplexers with $\mathrm{HE}_{11}$ resonators show high performance. A development of such a diplexer for $170 \mathrm{GHz}$, which is compatible with the ITER ECRH system is underway.

\section{References}

1. W. Kasparek et al., Fusion Eng. Design 84 (2009) 1002-1005

2. M. I. Petelin, AIP Conference Proceedings 691 (2003) 251-262

3. M. A. Henderson et al., Nucl. Fusion 48 (2008) 054013

4. M. Maraschek, et al., Phys. Rev. Lett. 98 (2007) 025005

5. W.A. Bongers et al., Fusion Sci. Technol. 55 (2009) 188-203

6. W. Kasparek et al., Fusion Sci Technol. 59 (2011) 729 - 741

7. N. Doelman, et al., this conference digest

8. V. Erckmann et al., Fusion Sci. Technol. 52 (2007) 291

9. D. Wagner et al., Fusion Sci. Technol. 52 (2007) 313 - 320

10. W. Bongers et al., this conference digest

11. W. Kasparek, et al., Proc. of 16th Int. Workshop EC-16, Sanya, China, 2010. 